Slagter, S., et al., 2020, Experimental evidence supports early silica cementation of the Ediacara Biota: Geology, v. 49, https://doi.org/10.1130/G47919.1

\title{
Experimental evidence supports early silica cementation of the Ediacara Biota
}

Silvina Slagter ${ }^{1}$, Lidya G. Tarhan ${ }^{1}$, Weiduo $\mathrm{Hao}^{2}$, Noah J. Planavsky ${ }^{1}$, and Kurt O. Konhauser ${ }^{2}$

${ }^{1}$ Department of Earth and Planetary Sciences, Yale University, New Haven, CT 06511, USA

${ }^{2}$ Department of Earth and Atmospheric Sciences, University of Alberta, Edmonton, AB T6G 2E3, Canada

Pg. 2-3: Methods.

Pg. 10: References cited.

Table S1: pH measurements.

Table S2: List of ingredients for silica assay.

Table S3: Sample characteristics.

Table S4: Log Ka and site density results.

Figure S1: Images of Marsilea and Quercus specimens through time.

Figure S2: Acid-base titration curve for Cassiopea.

Figure S3: Acid-base titration curve for Phymantus.

Figure S4: SEM images of Cinachyra spicules through time. 


\section{METHODS}

\section{Experimental Materials}

Carolina Biological Supply Company (Burlington, NC, USA) product numbers:

Cinachyra alloclada (Prod. No. \#162927)

Cassiopea (Prod. No. \#162936)

Phymanthus crucifer (Prod. No. \#162942)

Marsilea (Prod. No. \#156931)

Spirulina (Prod. No. \#151900)

\section{Water and tissue sampling}

Water samples $(2 \mathrm{~mL})$ were removed by syringe at various intervals and stored in tightly capped polyethylene containers for analysis of silica concentration. Part $(0.2 \mathrm{ml})$ of each sample was separated to evaluate $\mathrm{pH}$ change over time using serological pipettes (Table DR1). Solution $\mathrm{pH}$ was measured using a Thermo Scientific ${ }^{\mathrm{TM}}$ Orion $^{\mathrm{TM}}$ PerpHecT ${ }^{\mathrm{TM}}$ ROSS $^{\mathrm{TM}}$ Combination $\mathrm{pH}$ Micro Electrode, calibrated with the $\mathrm{pH}$ buffers 4.0, 7.0, 10.0 before each analysis. The error associated with the $\mathrm{pH}$ measurement is within $\pm 0.02 \log$ units $(2 \sigma)$. Organic tissues were also sampled at the same intervals for scanning electron microscope and energy-dispersive x-ray spectroscopy (SEM-EDS) observations. Concentrations of silica were measured by the molybdate blue method with metol as the reducing agent (Mullin and Riley, 1955) at $812 \mathrm{~nm}$ using a UV/VIS spectrophotometer (Table DR2).

Organic tissue samples were fixed in $4 \%$ paraformaldehyde, stored at $4^{\circ} \mathrm{C}$, and then coated with $6 \mathrm{~nm}$ platinum for SEM-EDS analysis with a Secondary Electron (SE) detector. SEM-EDS analyses were performed at 3-10 kV with a FEI XL-30 scanning electron microscope and a Hitachi SU7000 in the Department of Earth and Planetary Sciences at Yale University. Specimen dimensions and weights are detailed in Table DR3. 


\section{Acid-base titrations}

Potentiometric acid-base titrations were performed at the Department of Earth and Atmospheric Sciences at the University of Alberta. Analyses were performed for each tissue type (5 samples of each) to determine their proton reactivities. Before each titration, the $\mathrm{pH}$ electrode was calibrated using commercial $\mathrm{pH}$ buffers (Thermo Fisher Scientific; $\mathrm{pH}$ 4.0, 7.0, 10.0). For each titration, organic tissues were suspended in a $0.56 \mathrm{M} \mathrm{NaCl}$ solution. The suspension was then bubbled for 30 min with $\mathrm{N}_{2}(\mathrm{~g})$ to ensure the solution was devoid of $\mathrm{CO}_{2}$. During titrations, the experimental apparatus remained sealed and was continuously bubbled with $\mathrm{N}_{2}$ to prevent $\mathrm{CO}_{2}$ from entering the system. Each organic tissue sample was titrated over a pH range of 3.0 to 10.5. Initially, a small volume of $0.1 \mathrm{M}$ nitric acid $\left(\mathrm{HNO}_{3}, \mathrm{ACS}\right.$ certified, Fisher Scientific) was added to bring $\mathrm{pH}$ to 3.0 , and then $0.1 \mathrm{M}$ sodium hydroxide $(\mathrm{NaOH}$, ACS certified, Fisher Scientific) solution was incrementally added to bring the $\mathrm{pH}$ up to 10.5 (forward titration). To test the hysteresis of the samples, a backward titration was performed after each forward titration by adding acid to decrease the solution $\mathrm{pH}$ from 10.5 to 3.0. After the volume of acid and base were added, the corresponding $\mathrm{pH}$ changes were recorded at each titration step. The $\mathrm{pH}$ was considered stable only after the electrode achieved a reading of $12 \mathrm{mV} / \mathrm{min}$. A blank titration, without the addition of biomass, was performed for electrolyte solutions at each of the titrations performed.

Surface complexation modeling was performed for acid-base titration results of organic tissue samples. Three types of surface functional groups $(\equiv \mathrm{LH}, \equiv \mathrm{XH}, \equiv \mathrm{MH})$ were invoked to represent surface proton-reactive groups. The protonation behaviors of the three surface sites are described as follows:

$$
\begin{aligned}
& \equiv \mathrm{L}^{-}+\mathrm{H}^{+} \leftrightarrow \equiv \mathrm{LH} \\
& \mathrm{K}_{\mathrm{a} 1}=\frac{[\equiv \mathrm{LH}]}{\left[\equiv \mathrm{L}^{-}\right] \bullet \alpha_{\mathrm{H}^{+}}} \\
& \equiv \mathrm{X}^{-}+\mathrm{H}^{+} \leftrightarrow \equiv \mathrm{XH} \\
& \mathrm{K}_{\mathrm{a} 2}=\frac{[\equiv \mathrm{XH}]}{\left[\equiv \mathrm{X}^{-}\right] \bullet \alpha_{\mathrm{H}^{+}}} \\
& \equiv \mathrm{M}^{-}+\mathrm{H}^{+} \leftrightarrow \equiv \mathrm{MH}
\end{aligned}
$$




$$
\mathrm{K}_{\mathrm{a} 3}=\frac{[\equiv \mathrm{MH}]}{\left[\equiv \mathrm{M}^{-}\right] \bullet \alpha_{\mathrm{H}^{+}}}
$$

where square brackets denote the concentration of surface functional groups and $\alpha_{\mathrm{H}^{+}}$the activity of protons in solution; $\mathrm{K}$ values derived by equations 1-3 are proton interaction constants that govern the adsorption and desorption of protons from the sample's surfaces.

A surface complexation model (SCM) was generated using the titration data and the modeling software FITEQL 4.0 (Westall, 1982) in order to calculate $\mathrm{K}_{\mathrm{a}}$ values and ligand concentrations that best describe the excess charge data. The initial protonation stage was estimated based on Fein et al. (2005).

Table S1. $\mathrm{pH}$ measurements

\begin{tabular}{c|c|cccccccccccc}
\hline \hline Hour & & 0 & 1 & 2 & 18 & 26 & 48 & 97 & 117 & 121 & 141 & 144 & 148 \\
\hline \multirow{3}{*}{ Cassiopea } & 1 & 7.8 & 7 & 7.8 & 7 & 6.7 & 6.7 & 7.8 & 7.8 & 7.8 & 7.5 & 7.4 & 7.7 \\
& 2 & 7.8 & 7.7 & 7.7 & 7.8 & 8 & 7.8 & 7.7 & 7.7 & 7.7 & 7.7 & 7.5 & 7.7 \\
& 3 & 7.7 & 7.7 & 7.6 & 7.6 & 7.6 & 7.6 & 7.6 & 7.5 & 7.7 & 7.5 & 7.4 & 7.4 \\
\hline \multirow{4}{*}{ Cynachira } & 1 & 7.8 & 7.1 & 6.9 & 6.8 & 6.9 & 6.7 & 7.6 & 6.8 & 6.9 & 7.2 & 7.7 & 7.3 \\
& 2 & 7.8 & 7.8 & 7.8 & 7.8 & 7.8 & 7.8 & 7.8 & 7.8 & 7.8 & 7.8 & 7.8 & 7.2 \\
& 3 & 7.8 & 7.8 & 7.8 & 7.8 & 7.8 & 7.8 & 7.7 & 7.8 & 7.8 & 7.6 & 7.6 & 7.8 \\
\hline \multirow{3}{*}{ Spirulina } & 1 & 7.8 & 7.8 & 7.8 & 7.8 & 7.8 & 7.8 & 7.8 & 7.8 & 7.8 & 7.8 & 7.8 & 7.8 \\
& 2 & 7.8 & 7.8 & 7.8 & 7.8 & 7.8 & 7.8 & 7.8 & 7.8 & 7.8 & 7.8 & 7.8 & 7.8 \\
& 3 & 7.8 & 8 & 7.6 & 7.8 & 7.1 & 7.8 & 7.8 & 7.8 & 7.8 & 7.8 & 7.8 & 7.8 \\
\hline \multirow{3}{*}{ Marsilea } & 1 & 7.8 & 7.8 & 7.8 & 7.8 & 7.8 & 7.8 & 7.8 & 7.8 & 7.8 & 7.8 & 7.8 & 7.8 \\
& 2 & 7.8 & 7.8 & 7.7 & 7.8 & 7.6 & 7.8 & 7.8 & 7.7 & 7.8 & 7.8 & 7.8 & 7.8 \\
& 3 & 7.8 & 7.8 & 7.8 & 7.8 & 7.8 & 7.8 & 7.8 & 7.8 & 7.8 & 7.8 & 7.8 & 7.8 \\
\hline & 1 & 7.8 & 7.8 & 7.8 & 7.8 & 7.8 & 7.8 & 7.8 & 7.8 & 7.8 & 7.8 & 7.8 & 7.8 \\
& 2 & 7.8 & 7.8 & 7.8 & 7.8 & 7.7 & 7.8 & 7.8 & 7.8 & 7.8 & 7.8 & 7.8 & 7.8 \\
& 3 & 7.8 & 7.8 & 7.8 & 7.8 & 7.8 & 7.8 & 7.8 & 7.8 & 7.8 & 7.8 & 7.8 & 7.8 \\
\hline \multirow{3}{*}{ Quercus } & 1 & 7.8 & 7.8 & 7.8 & 7.8 & 7.7 & 7.8 & 7.8 & 7.8 & 7.8 & 7.8 & 7.8 & 7.8 \\
& 2 & 7.8 & 7.8 & 7.8 & 7.8 & 7.8 & 7.8 & 7.8 & 7.8 & 7.8 & 7.8 & 7.8 & 7.8 \\
& 3 & 7.8 & 7.8 & 7.8 & 7.8 & 7.8 & 7.8 & 7.8 & 7.8 & 7.8 & 7.8 & 7.8 & 7.8 \\
\hline \multirow{3}{*}{ control } & 1 & 7.8 & 7.8 & 7.8 & 7.8 & 7.8 & 7.8 & 7.8 & 7.8 & 7.8 & 7.8 & 7.7 & 7.8 \\
& 2 & 7.8 & 7.8 & 7.8 & 7.8 & 7.8 & 7.8 & 7.8 & 7.8 & 7.8 & 7.8 & 7.8 & 7.7 \\
& 3 & 7.8 & 7.8 & 7.8 & 7.8 & 7.8 & 7.8 & 7.7 & 7.8 & 7.8 & 7.6 & 7.6 & 7.7 \\
\hline
\end{tabular}


Table S2: List of ingredients for silica assay*

$$
\begin{gathered}
\text { Concentration }(\mathrm{g} \text { solute } / 500 \mathrm{~mL} \\
\mathrm{dd \textrm {H } _ { 2 } \mathrm { O } )}
\end{gathered}
$$

Molybdate reagent

Ammonium molybdate $\quad 4$

$12 \mathrm{~N}$ Hydrochloric acid $\quad 12.0$

$\underline{\text { Reducing reagent }}$

Metol-sulfite

Anhydrous sodium sulfite $\quad 6$

P-methyl aminophenol sulfate $\quad 10$

Oxalic acid solution $\quad 50$

Sulfuric acid $(50 \%) \quad 250.0^{\dagger}$

Note: The molybdate reagent, metol-sulfite, oxalic acid and sulfuric acid solutions were prepared independently in $500 \mathrm{~mL}$ of $\mathrm{ddH}_{2} \mathrm{O}$. Metol-sulfite, oxalic acid and sulfuric acid were mixed together to form the reducing agent (5:3:3, respectively, $\mathrm{v} / \mathrm{v} / \mathrm{v}) .2 \mathrm{~mL}$ of molybdate reagent and $3 \mathrm{~mL}$ of reducing reagent were added to $4 \mathrm{~mL}$ of sample before analyzing silica concentrations. Blanks and standard were prepared by the addition of $0.2 \mathrm{ml}$ of artificial seawater to $3.8 \mathrm{ml}$ of initial sample.

* Modified from Newman et al. (2016)

$\dagger$ Represents the addition of a liquid volume (in $\mathrm{mL}$ ).

Table S3. Sample dimension, weight and time of decay

\begin{tabular}{|c|c|c|c|c|}
\hline Sample & & $\begin{array}{l}\text { Diameter } \\
(\mathrm{cm})\end{array}$ & Weight $(\mathrm{g})$ & $\begin{array}{c}\text { Time to complete } \\
\text { decay }(\mathrm{h})\end{array}$ \\
\hline \multirow{3}{*}{ Cassiopea } & 1 & 7.1 & 5.09 & 130 \\
\hline & 2 & 7.2 & 6.21 & 125 \\
\hline & 3 & 6.8 & 4.89 & 125 \\
\hline \multirow{3}{*}{ Cynachira } & 1 & 6.8 & 6.88 & N.D* \\
\hline & 2 & 5.2 & 5.77 & N.D* \\
\hline & 3 & 6.8 & 5.11 & N.D* \\
\hline \multirow{3}{*}{ Spirulina } & 1 & 0.5 & 1.023 & 132 \\
\hline & 2 & 0.3 & 2.33 & 130 \\
\hline & 3 & 0.25 & 0.23 & 123 \\
\hline \multirow{3}{*}{ Marsilea } & 1 & N.D* & 5.3 & N.D* \\
\hline & 2 & N.D* & 8 & N.D* \\
\hline & 3 & N.D* & 7.23 & N.D* \\
\hline \multirow{3}{*}{ Phymantus } & 1 & 1.2 & 4 & 142 \\
\hline & 2 & 1.5 & 4.8 & 147 \\
\hline & 3 & 2 & 5 & 150 \\
\hline \multirow{3}{*}{ Quercus } & 1 & N.D* & 2.3 & N.D* \\
\hline & 2 & N.D* & 2.2 & N.D* \\
\hline & 3 & N.D* & 2.1 & N.D* \\
\hline
\end{tabular}

*N.D: not determined 
Table S4. Summary of acid-base titration and SCM results and comparison with literature results. Site density and $\mathrm{pKa}$ values represent the average values of all replicates.

\begin{tabular}{|c|c|c|c|c|}
\hline & $\begin{array}{l}\text { Ligand } \\
\text { class }\end{array}$ & Average $\mathrm{pKa}$ & $\begin{array}{c}\text { Mean site concentration } \\
(\mathrm{mol} / \mathrm{g})\end{array}$ & Functional group ${ }^{a}$ \\
\hline \multirow{3}{*}{ Cassiopea } & LH & 4.09 & $8.01 \mathrm{E}-06$ & Carboxyl \\
\hline & $\mathrm{XH}$ & 6.41 & $3.69 \mathrm{E}-06$ & Carboxyl or phosphoryl \\
\hline & MH & 9.57 & $3.45 \mathrm{E}-05$ & Amino \\
\hline \multirow{3}{*}{ Cynachira } & LH & 4.48 & $2.16 \mathrm{E}-04$ & Carboxyl \\
\hline & $\mathrm{XH}$ & 6.49 & $2.62 \mathrm{E}-05$ & Carboxyl or phosphoryl \\
\hline & MH & 9.59 & $3.18 \mathrm{E}-04$ & Amino \\
\hline \multirow{3}{*}{ Spirulina } & LH & 6.37 & $1.33 \mathrm{E}-05$ & Carboxyl or phosphoryl \\
\hline & $\mathrm{XH}$ & 9.1 & $6.26 \mathrm{E}-04$ & Amino \\
\hline & MH & 10.39 & $1.06 \mathrm{E}-04$ & Amino \\
\hline \multirow{3}{*}{ Marsilea } & LH & 4.22 & $1.39 \mathrm{E}-04$ & Carboxyl \\
\hline & $\mathrm{XH}$ & 6.47 & $3.76 \mathrm{E}-05$ & Carboxyl or phosphoryl \\
\hline & MH & 9.62 & $6.41 \mathrm{E}-05$ & Amino \\
\hline \multirow{3}{*}{ Phymantus } & LH & 4.48 & 4.04E-04 & Carboxyl \\
\hline & $\mathrm{XH}$ & 7.18 & $2.64 \mathrm{E}-05$ & Phosphoryl \\
\hline & $\mathrm{MH}$ & 9.64 & 4.57E-04 & Amino \\
\hline \multirow{3}{*}{ Quercus } & LH & 5.14 & $3.25 \mathrm{E}-05$ & Carboxyl \\
\hline & $\mathrm{XH}$ & 7.37 & 4.44E-05 & Phosphoryl \\
\hline & MH & 9.4 & $8.53 \mathrm{E}-05$ & Amino \\
\hline \multicolumn{5}{|c|}{ Literature results for biomass titration and SCM } \\
\hline \multirow{3}{*}{$\begin{array}{l}\text { Calothrix isolated } \\
\text { sheath }^{\mathrm{b}}\end{array}$} & LH & 4.62 & $3.30 \mathrm{E}-05$ & Carboxyl \\
\hline & $\mathrm{XH}$ & $6.12-7.26$ & $1.4-2.7 \mathrm{E}-05$ & Carboxyl or phosphoryl \\
\hline & MH & $8.06-9.15$ & 2.3-4.1E-05 & Amino \\
\hline \multirow{3}{*}{ Worm mucus $^{\mathrm{c}}$} & LH & 4.89 & $5.20 \mathrm{E}-03$ & Carboxyl \\
\hline & $\mathrm{XH}$ & 6.93 & $3.55 \mathrm{E}-03$ & Phosphoryl \\
\hline & MH & 8.98 & $2.51 \mathrm{E}-03$ & Thiol \\
\hline \multirow{3}{*}{ Worm mucus $^{\mathrm{d}}$} & LH & 4.82 & $5.73 \mathrm{E}-03$ & Carboxyl \\
\hline & $\mathrm{XH}$ & 7.08 & 4.67E-03 & Phosphoryl \\
\hline & $\mathrm{MH}$ & 9.3 & $6.89 \mathrm{E}-03$ & Thiol or amino \\
\hline \multirow{3}{*}{$\begin{array}{l}\text { Synechococcus sp. } \\
\text { PCC } 7002^{\mathrm{e}}\end{array}$} & LH & $5.07-5.42$ & $1.17-1.51 \mathrm{E}-02$ & Carboxyl \\
\hline & $\mathrm{XH}$ & $6.71-7.42$ & $5.72-6.55 \mathrm{E}-03$ & Phosphoryl \\
\hline & MH & $8.54-9.95$ & $6.96-11.07 \mathrm{E}-03$ & Amino \\
\hline Alginate $^{f}$ & LH & 3.98 & $1.73 \mathrm{E}-03$ & Carboxyl \\
\hline $\begin{array}{l}\text { Diopatra } \\
\text { parchment }\end{array}$ & $\mathrm{MH}$ & -1.079613993 & $1.17 \mathrm{E}-05$ & Hydroxyl \\
\hline
\end{tabular}


${ }^{\mathrm{d}}$ results from Petrash et al. (2011a)

${ }^{\mathrm{e}}$ results from Liu et al. (2015)

${ }^{\mathrm{f}}$ results from Petrash et al. (2011b)

${ }^{\mathrm{g}}$ results from Konhauser et al. (2020)

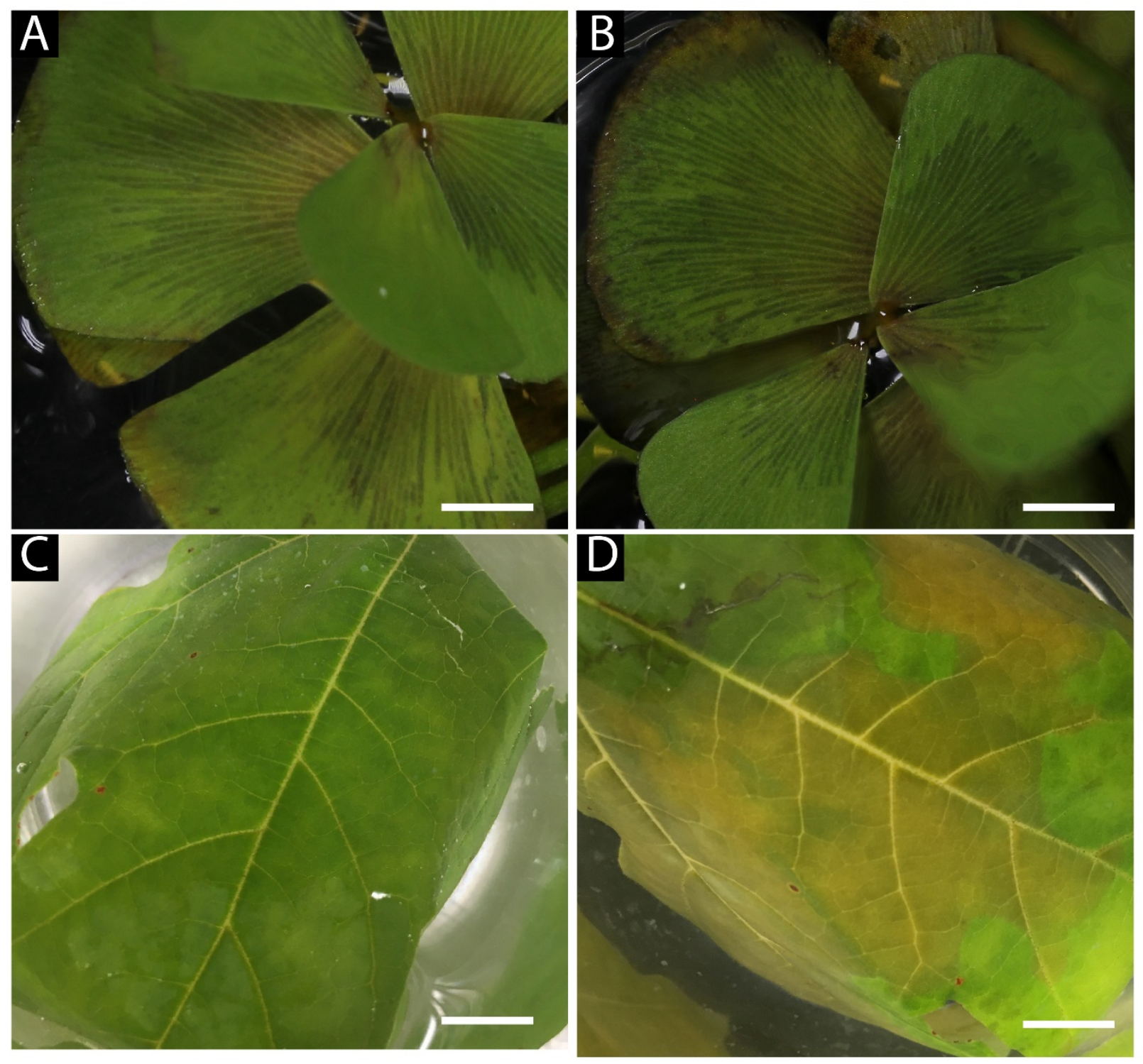

Figure S1. Images of Marsilea in experiments after: (A) 60 h, and (B) 120 h. Images of Quercus in experiments after: (C) $24 \mathrm{~h}$, and (D) $96 \mathrm{~h}$. Note lack of visible signs of silica precipitation. Scale bar is $2 \mathrm{~mm}$. 


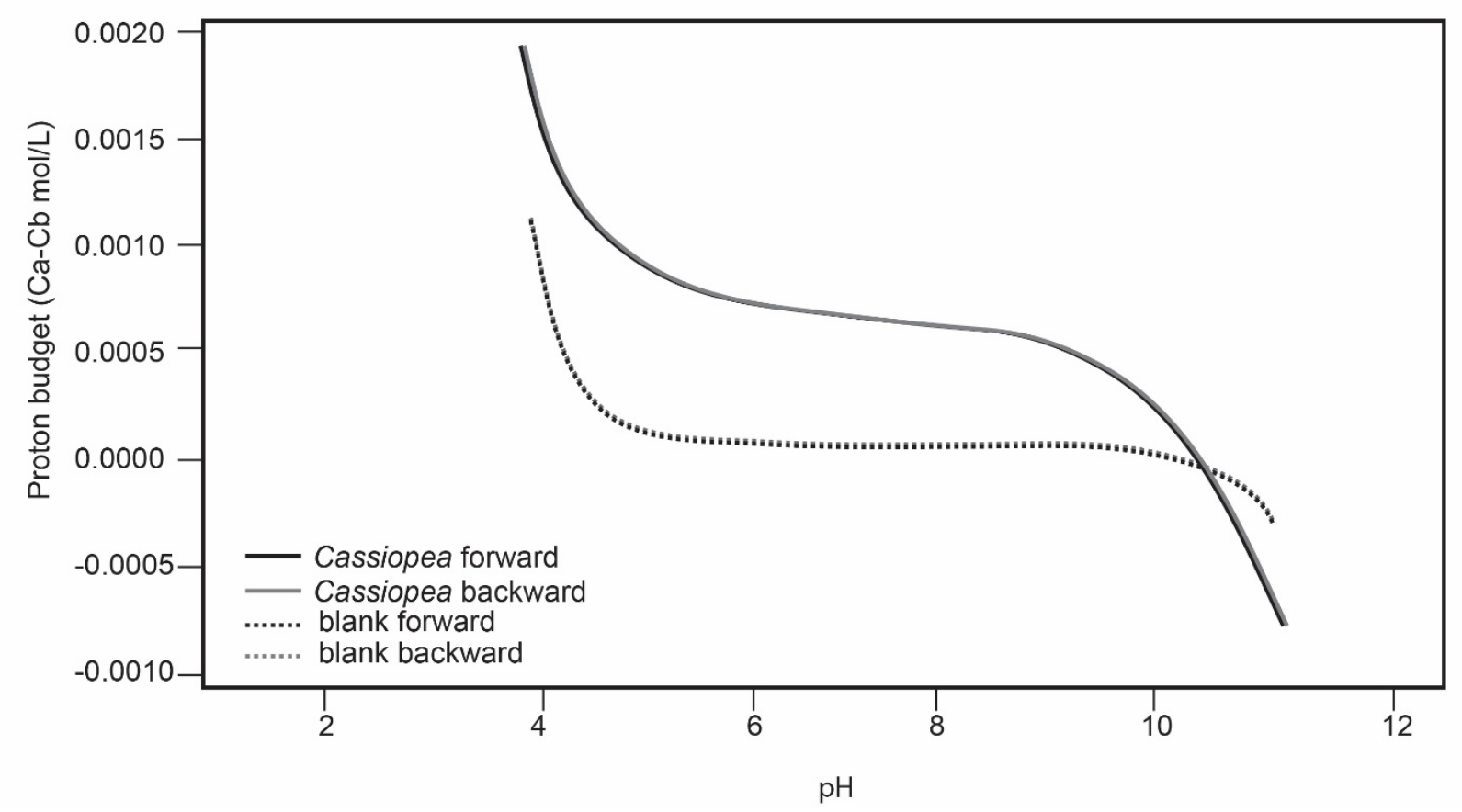

Figure S2. Acid-base titration curves for Cassiopea.

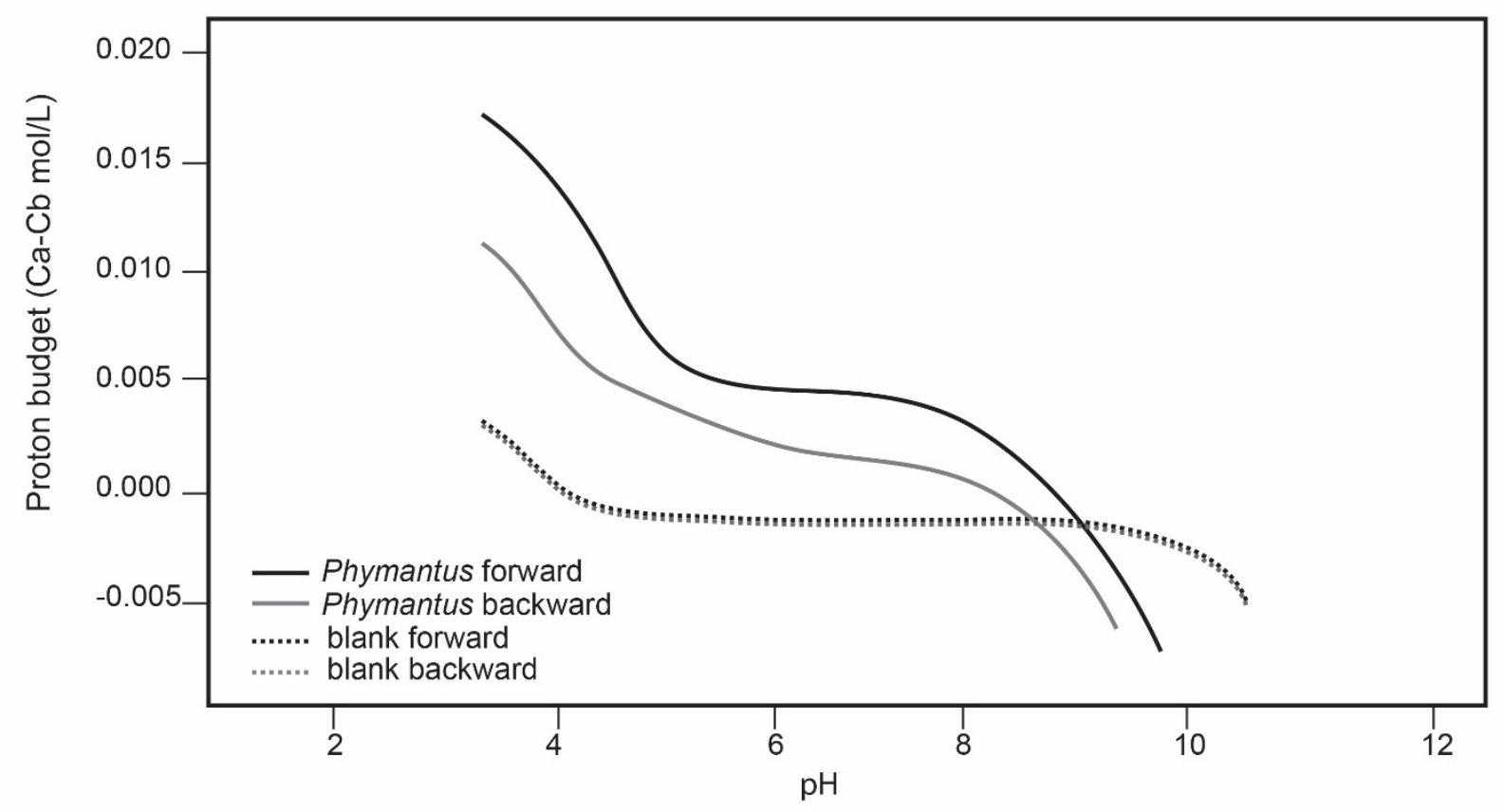

Figure S3. Acid-base titration curves for Phymantus. 

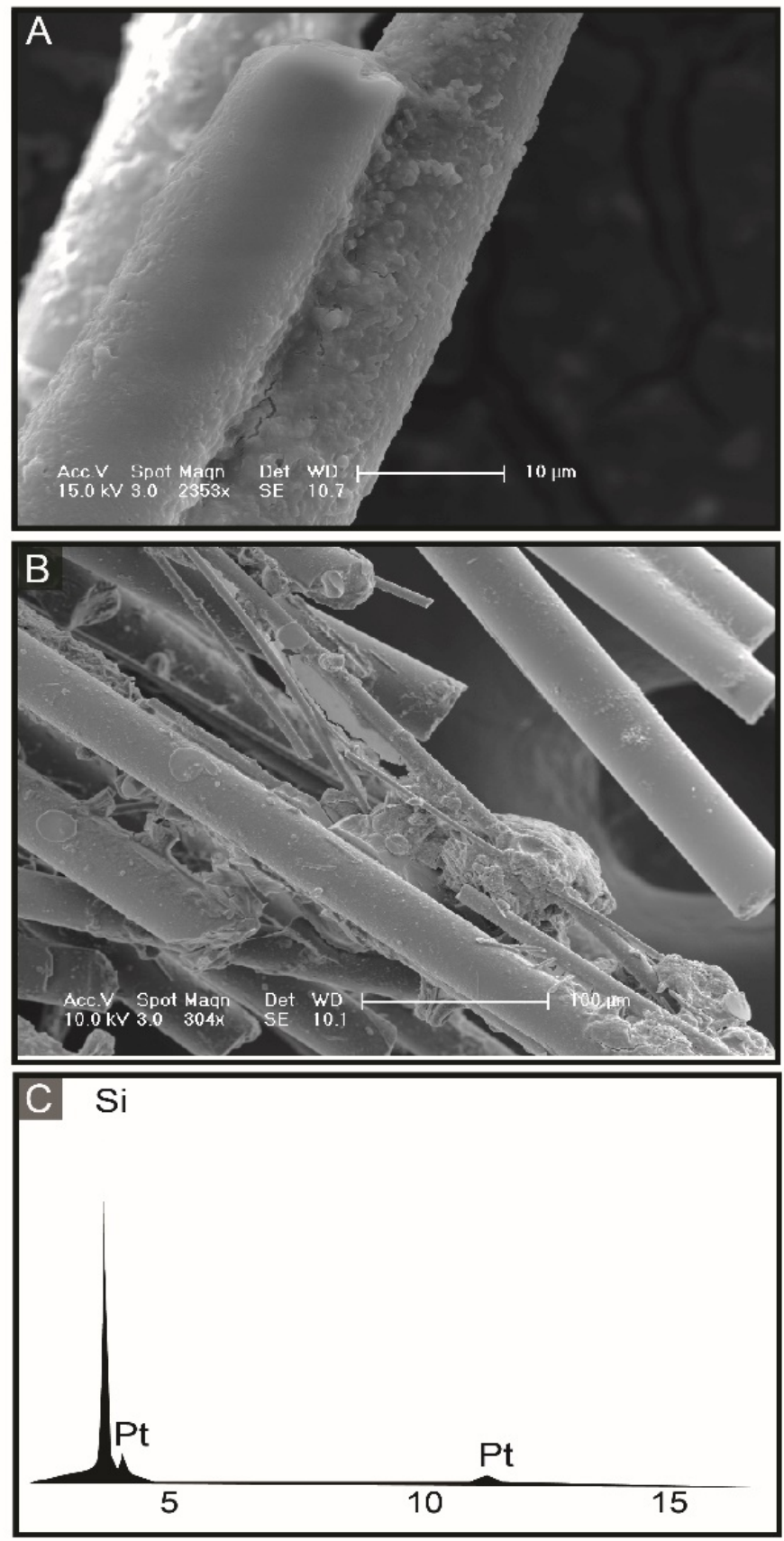

Figure S4. SEM images of pristine demosponge spicules in experiments after (A) 60 hours, and (B) 100 hours. (C) EDS spectra of panel B demonstrating the siliceous composition of the spicules. Pt peaks correspond to the coating. 


\section{REFERENCES CITED}

Fein, J.B., Boily J.-F., Yee, N., Gorman-Lewis, D., Turner, B.F., 2005, Potentiometric titrations of Bacillus subtilis cells to low $\mathrm{pH}$ and a comparison of modeling approaches: Geochimica, et Cosmochimica, Acta, v. 69, p. 1123-1132, https://doi.org/10.1016/j.gca.2004.07.033.

Hao, W., Flynn, S.L., Kashiwabara, T., Alam, M.S., Bandara, S., Robbins, L.J., Alessi, D.S., Konhauser, K.O., 2019, The impact of ionic strength on the proton reactivity of clay minerals: Chemical Geology, v. 529, p. 1192-94, https://doi.org/10.1016/j.chemgeo.2019.119294.

Konhauser, K.O., 2009, Introduction to geomicrobiology, John Wiley \& Sons, Chapter 3, p. 102.

Konhauser, K.O., Hao, W., Li, Y., von Gunten, K., Bishop, B.A., Alessi, D.S., Tarhan, L.G., O'Connell, B., Robbins, L.J., Planavsky, N.J. and Gingras, M.K., 2020, Diopatra cuprea worm burrow parchment: a cautionary tale of infaunal surface reactivity: Lethaia, https://doi.org/10.1111/let.12335.

Lalonde, S.V., Dafoe, L.T., Pemberton, S.G., Gingras, M.K., Konhauser, K.O., 2010, Investigating the geochemical impact of burrowing animals: Proton and cadmium adsorption onto the mucus lining of Terebellid polychaete worms: Chemical geology, v. 271, p. 44-51, https://doi.org/10.1016/j.chemgeo.2009.12.010

Liu, Y., Alessi, D.S., Owttrim, G.W., Petrash, D.A., Mloszewska, A.M., Lalonde, S.V., Martinez, R.E., Zhou, Q., Konhauser, K.O., 2015, Cell surface reactivity of Synechococcus sp. PCC 7002: Implications for metal sorption from seawater: Geochimica et Cosmochimica Acta, v., 169, p. 30-44, doi: https://doi.org/10.1016/j.gca.2015.07.033.

Newman, S.A., Mariotti, G., Pruss, S., \& Bosak, T., 2016, Insights into cyanobacterial fossilization in Ediacaran siliciclastic environments: Geology, v. 44, p. 579-582, https://doi.org/0.1130/G37791.1

Mullin J.B., Riley J.P., 1955, The colorimetric determination of silicate with special reference to sea and natural waters: Analytica Chimica Acta, v. 12, p. 162-176. https://doi.org/10.1016/S0003-2670(00)87825-3 
Petrash, D.A., Lalonde, S.V., Gingras, M.K., Konhauser, K.O., 2011a, A surrogate approach to studying the chemical reactivity of burrow mucous linings in marine sediments: Palaios, v. 26, p. 594-600, https://doi.org/10.2110/palo.2010.p10-140r

Petrash, D.A., Lalonde, S.V., Raudsepp, M., Konhauser, K.O., 2011b, Assessing the importance of organic matrix materials in biofilm chemical reactivity: insights from proton and cadmium adsorption onto the commercially available biopolymer alginate: Geomicrobiology Journal, v. 28, p. 266-273.

Phoenix, V. R., Martinez, R.E., Konhauser, K.O., and Ferris, F.G., 2002, Characterization and Implications of the Cell Surface Reactivity of Calothrix sp. Strain KC97: Applied and $\begin{array}{lllll}\text { environmental microbiology, } & \text { v. } & 68, & \text { p. }\end{array}$ https://doi.org/10.1128/AEM.68.10.4827-4834.2002.

Westall, J.C., 1982. FITEQL: A Computer Program for Determination of Chemical Equilibrium Constants from Experimental Data. Department of Chemistry, Oregon State University. 